\title{
Advanced Prediction Based Mobility Support for 6LoWPAN Wireless Sensor Networks
}

\author{
Riaz Ahmed Khan \\ National Institute of Technology/ECE, Srinagar, 190006, India \\ E-mail: riazk3@gmail.com \\ Ajaz Hussain Mir \\ National Institute of Technology/ECE, Srinagar, 190006, India \\ E-mail: ahmir@rediffmail.com
}

\begin{abstract}
Wireless Sensor Nodes (SNs), the key elements for building Internet of Things (IOT) have been deployed widely in order to get and transmit information over the internet. IPv6 over low power personal area network (6LoWPAN) enabled their connectivity with IPV6 networks. 6LoWPAN has mobility and it can find an extensive application space only if provides mobility support efficiently. Existing mobility schemes are focused on reducing handoff (HO) latency and pay less attention towards packet loss and signaling cost. In time critical applications under IOT, packet loss and excessive signaling cost are not acceptable. This paper proposes a scheme based on advanced mobility prediction for reducing extra signaling cost and packet loss that incurs due to connection termination in traditional schemes such as Proxy Mobile IPv6 (PMIPv6) handover. In our proposed scheme 6LoWPAN WSN architecture with IPv6 addressing is presented. Based on this architecture the mobility algorithm is proposed for reducing signaling cost, packet loss by buffering mechanism and HO latency in particular. In the algorithm layer 2 (L2) and layer 3 (L3) $\mathrm{HO}$ is performed simultaneously with prior $\mathrm{HO}$ prediction with no Care of Address (CoA) configuration which also reduces signaling cost to some extent. The proposed scheme is analyzed theoretically and evaluated for different performance metrics. Data results showed significant improvements in reducing packet loss, signaling cost and HO latency when compared to standard PMIPv6 in time critical scenarios.
\end{abstract}

Index Terms-IP Mobility, PMIPv6 (Proxy Mobile IPv6), Handoff latency, Packet Loss, 6LoWPAN (IPv6 enabled low power personal area networks), IOT (Internet of Things).

\section{INTRODUCTION}

With the emergence of IOT [1] and machine to machine communication (M2M) [2], the connectivity of wireless sensor networks (WSN) to the internet has become very urgent. Wireless sensor nodes can sense physical parameters, gather information and transmit it on the network. IPv6 [RFC 4291] provides enough address space to locate these numerous resource constrained devices and 6LoWPAN [RFC 4919] enables their connectivity with IPv6 networks. 6LoWPAN has mobility and therefore it can find a wide application area if supported by an efficient mobility scheme. Mobility in 6LoWPAN WSN has been approached in different perspectives to target varied applications including healthcare, disaster management, security surveillance, military operations and intelligent transport system (ITS)

[3]. Mobility protocols have come over time with growth of mobile devices and find much interest in 6LoWPAN networks. These protocols are classified into the Host-Based Schemes [4] and the Network-Based Schemes [5]. In host-based schemes, Mobile Sensor Node (MSN) has to be involved in signaling process which includes exchange of Binding Update (BU) and Binding Acknowledgement (BA) messages for Care of Address (CoA) configuration [6,7]. On the other hand, in networkbased schemes MSN is relieved from heavy mobility signaling, instead network components are responsible for detecting its movement and address configuration [8]. Researchers in the field have used both types of protocols with different perspectives to improve the $\mathrm{HO}$ performance in 6LoWPAN WSN. Most of the leading solutions in [9-13] are PMIPv6 based, focus on reducing HO latency only and pay less attention towards packet loss and signaling cost. The packet loss incurs due to heavy signaling cost and connection termination in PMIPv6-HO, is unacceptable in time critical applications [3]. During PMIPv6-HO, connection terminates and packets sent to Previous Access Router (PAR) are lost as $\mathrm{MN}$ moves to new domain before PAR gets notified about HO. The heavy signaling cost causes longer registration delay and packet loss which results in information loss. Although these mobility solutions improve $\mathrm{HO}$ performance in 6LoWPAN networks to some extent based either on L2 HO or L3 HO independently but do not combine them efficiently. Therefore, L2 HO is performed followed by L3 HO which results in degradation of $\mathrm{HO}$ performance to some extent.

To improve the mobility HO in 6LoWPAN networks, this paper proposes an advanced mobility prediction based scheme for 6LoWPAN networks with following contributions: 
1). A 6LoWPAN WSN architecture with IPv6 addressing in distributed fashion is presented. In this addressing scheme a node can acquire its unique address from its associate node thus constructing a tree called routing backbone. For routing of packets, globally identified network prefix called Home Network Prefix (HNP) of communicating node is used. Further in the domain, packets received are sent to respective or desired interface ID.

2). Based on this architecture advanced mobility prediction scheme is proposed. In this scheme $\mathrm{HO}$ can be predicted by checking connection quality strength. A connection quality comparison algorithm is also presented. In the proposed scheme New Access Router (NAR) can be triggered for $\mathrm{HO}$ prior to actual disconnection from previous link thus saves registration time. The NAR will buffer the traffic until the mobile node (MN) is connected after HO or PAR will buffer the traffic if $\mathrm{MN}$ is already disconnected and then redirect buffered traffic to NAR. This buffering mechanism prevents the packet loss and as a result HO performance is improved.

3). L2 and L3 HO is performed simultaneously without channel scanning and configuring CoA. For L2 HO, L2 info-message is used and for L3 HO no CoA is configured. As a result $\mathrm{HO}$ latency, signaling cost and packet loss are reduced.

The remainder of this paper is organized as follows. Section 2 discusses the related work carried out on 6LoWPAN WSN mobility and in section 3, 6LoWPAN architecture with IPv6 addressing is presented. In section 4, the proposed scheme with mobility $\mathrm{HO}$ algorithm and its performance analysis is presented. In sections 5, proposed scheme is evaluated and results are discussed. Section 6 concludes the paper with its contributions.

\section{RELATED WORK}

The architecture differences and incompatibility issues between 6LoWPAN WSN and IPv6 networks lead to difficulties in actual deployment of such networks jointly. Due to which mobility solutions do not work efficiently in 6LoWPAN WSN and require further research [14]. Currently mobility solutions are classified into Hostbased schemes and network-based schemes. Host-based protocols such as Mobile IPv6 (MIPv6) [4] and Hierarchical MIPv6 (HMIPv6) [15] were initially designed for providing seamless mobility to MN. These protocols suffer heavy signaling cost due to exchange of BU and BA messages. Also these schemes were designed for wired networks due to which they cannot work efficiently in WSN [16]. Network-based mobility scheme such as PMIPv6 [5] is the only standardized scheme in the network-based category by internet engineering task force (IETF). PMIPv6 relieves the MN from heavy mobility signaling, instead network component called Mobile access gateway (MAG) performs signaling on behalf of MN. Mobility in resource constrained WSNs can efficiently be approached by PMIPv6 based solutions as MSN does not involve in mobility signaling. Researchers in the field have used both categories of protocols to achieve 6LoWPAN mobility.

A survey and comparison of mobility protocols is given in [17]. With focus on heterogeneous networks, a solution at data link layer, network layer and cross-layer is also proposed without host involvement concern. [18] highlighted host involvement in mobility and interoperability in IP level as major shortcomings of existing protocols. Therefore PMIPv6 was suggested to relieve the MN from heavy signaling. In [5], PMIPv6 is introduced and expected to support the real deployment of IP mobility management where $\mathrm{MN}$ is not supposed to be involved. In [19], ISMS is proposed which takes advantage of identity and location separation. It is intended to faster handover and route optimization. The ISMS HO delay is on the order of ms only which is far smaller than the traditional MIPv6. In [20], a scheme for 6LoWPAN dependent on dispatch type of the network is presented. Intra domain packet loss and HO delay were reduced but it is not suitable for inter domain 6LoWPANs Inter-Mario based on "make before break strategy" was proposed in [21] to support inter personal area network (Inter-PAN) HO in 6LoWPAN domain. In [22] a testbed based solution comprised of mobile and static gateways for soft $\mathrm{HO}$ of mobile WSN was proposed. The proposed SH-WSN6 was compared with MIH-PMIPv6, FPMIPv6 and PMIPv6. Also a Connection quality check algorithm to avoid unnecessary handover was presented but it lags in providing optimal threshold value. In [14], routing and mobility approaches for 6LoWPAN mesh networks is presented and also stated that the requirements and resources in the existing solutions for 6LoWPAN mobility is still a challenge. In [23], the mobility of wearable body sensors is addressed where reduced signal strength indicator (RSSI) is used to select the best AP. In [24], PMIPv6 to support IP mobility in different domains including WSNs is presented. SNs were used as LMA and MAG to carry out the mobility with same PMIPv6 operations. In [25], 6LoWMSN based on PMIPv6 was proposed to allow multi-hop communication in 6LoWPAN. Solutions in [26-28] are based on PMIPv6 protocol, addressing different 6LoWPAN WSN application areas for Improving $\mathrm{HO}$ performance. These solutions suffer heavy packet loss due to the connection termination in PMIPv6-HO. In [29], a solution based on network of proxies (NOP) is presented. NoP overlay on WSN and relives the SNs from signaling and processing tasks. NoP is found useful in energy consumption but use of additional structure which causes more expenditure is the major downside of it. Also extra HOs occur due to complex NOP structure is another problem. In [30], a cross-layer mobility supporting protocol which combines L2 and L3 HO is presented. The scheme improves the mobility $\mathrm{HO}$ performance by reducing $\mathrm{HO}$ latency but it does not reduce the packet loss completely and there is further scope of improvements.

More literature based on 6LoWPAN WSN mobility is available on different resources of literature. Although network-based schemes proved to be more efficient when 
compared to host-based schemes, still there is longer HO latency caused by address configuration, signaling cost due to exchange of BU and BA messages and packet loss due to connection termination. Therefore, 6LoWPAN WSN mobility is an open issue and requires more research in the field $[14,31]$.

\section{A. Our Solution:}

From the above discussions it can be seen that mobility solutions for 6LoWPAN WSN are well addressed by network-based schemes which improve HO performance to some extent. In these solutions, following are the factors which degrade the $\mathrm{HO}$ performance and are not acceptable in time critical scenarios.

1) L2 and L3 HO is performed separately. For L2 HO, channel scanning is the most time consuming process [34] whereas in $\mathrm{L} 3 \mathrm{HO}$, address configuration process contributes in degradation of HO performance.

2) During HO, there is connection termination between correspondent node $(\mathrm{CN})$ and $\mathrm{MN}$. Packets sent to PAR or NAR are lost which is not acceptable for time critical applications such as healthcare where any loss of packet can put patient's life in danger [27]. Also this packet loss can degrade the throughput which in turn degrades $\mathrm{HO}$ performance.

3) The host-based schemes involve $\mathrm{MN}$ in mobility signaling which SNs cannot manage as these are heavily resource constrained devices. Therefore host-based schemes are not useful in WSN.

Considering these issues this paper presents a mobility scheme for 6LoWPAN WSN with the following novelties to improve the $\mathrm{HO}$ performance:

1) A coordinating MSN can acquire its IP address from the associate node which contains network prefix and interface ID. For routing of packets, MSN is identified by HNP and within the domain, received packets are sent to desired interface ID.

2) L2 and L3 HO is performed simultaneously. Connection quality check algorithm to avoid unnecessary HOs and to prevent channel scanning is presented.

3) A buffering mechanism to prevent packet loss is also presented. During connection termination, buffering of packets is done either at PAR or NAR. After HO is completed buffered packets are delivered to MSN from NAR or they are redirected from PAR to NAR. This mechanism increases the throughput and hence the $\mathrm{HO}$ performance.

\section{6LOWPAN WSN ARCHITECTURE}

A 6LoWPAN WSN is mainly made up of two types of nodes: Fully-functional device (FFD) and Reducedfunctional device (RFD). FFDs are having the processing and routing functionalities whereas RFDs are only equipped with sensing or limited functionalities. FFDs can be fixed while as RFDs can be kept mobile. The link protocol used in 6LoWPAN is IEEE 802.15.4. In this protocol a 6LoWPAN can have one or multiple PANs. A PAN may consist of one gateway router, FFDs and RFDs. The PAN is connected to IPv6 networks through gateway access router. In the PAN, access router and FFDs make a routing backbone and each RFD has one associate node through which it can acquire its address. RFDs achieve communication through its associate node. Each node in the PAN is identified by HNP and uniquely identified by its interface ID. RFDs can be grouped and used for sensing different physical parameters in the PAN and their communication can be achieved through coordinating MSN. The 6LoWPAN WSN architecture is shown in fig. 1.

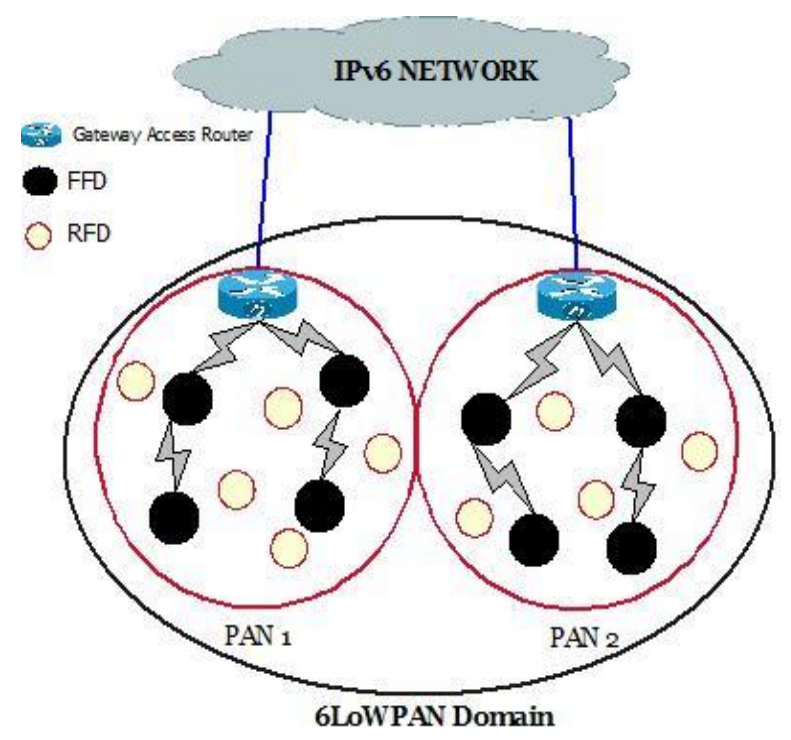

Fig.1. 6LoWPAN WSN Architecture

\section{A. Address Structure:}

The sensor nodes are usually distributed over a mobile object to collect physical parameters and these parameters can be transmitted through coordinating node. The architecture for address configuration is shown in fig. 2 . Based on this architecture, following address structure shown in table 1 is used.

Table 1. Address Structure

\begin{tabular}{|c|c|c|c|c|}
\hline \multicolumn{4}{|c|}{ Globally Identified Home Network Prefix (HNP) } & \\
\hline No. of Bits: & L & $\mathrm{m}$ & $\mathrm{n}$ & $(128-1-\mathrm{m}-\mathrm{n})$ bits \\
\hline Domain ID (Gateway AR) & Access Point (AP) ID & Coordinating Node ID & Interface ID \\
\hline
\end{tabular}


The address structure consists of four parts. First three parts constitute HNP and the last part is the node ID. Routing is achieved through HNP and then in the domain, packets are sent to the desired interface ID which uniquely identifies a node. The domain ID (l) is preset and the bits in AP ID (m), Coordinating Node ID (n) and interface ID (128-1-m-n) are determined depending upon network size and number of nodes in the domain.

In the architecture shown in fig. 2, each node acquires address from its associate node. Communication with the IPv6 networks is achieved through gateway access router. Initially the packets are received at gateway router and then sent to the desired interface ID within the PAN via APs.

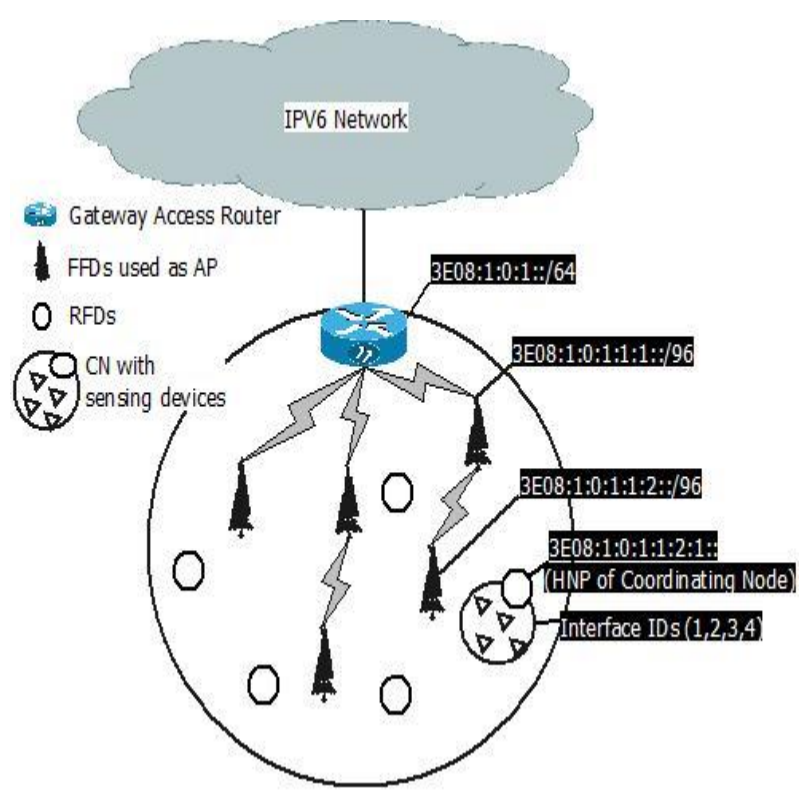

Fig.2. 6LoWPAN Address Structure

The maximum packet size for transmission in IEEE 802.15.4 is 127 bytes as the sensors are not capable of holding complete IPv6 address where packet size is 1280 bytes. 6LoWPAN adopts the IEEE 802.15.4 PHY, IEEE 802.15.4 MAC and Adaptation layers in its protocol stack [31]. The adaptation layer is responsible for fragmentation of packets, reassembly of fragments and IPv6 header compression.

\section{MOBILITY IN 6LOWPAN}

Mobility in 6LoWPAN is mainly supported by network-based protocols. Our solution is based on advanced HO prediction in network-based schemes to achieve prior registration before the actual disconnection of MSN from previous link. In order to know the HO imminence, signal strength generated by APs in the PAN is used. The AP with higher signal strength is selected and $\mathrm{HO}$ if required is initiated. Before $\mathrm{HO}$, a connection quality comparison check is done and depending upon the received signal strength (RSS) value, $\mathrm{HO}$ is made if another AP is found with higher RSS. The algorithm for connection quality check is given in fig. 3 .

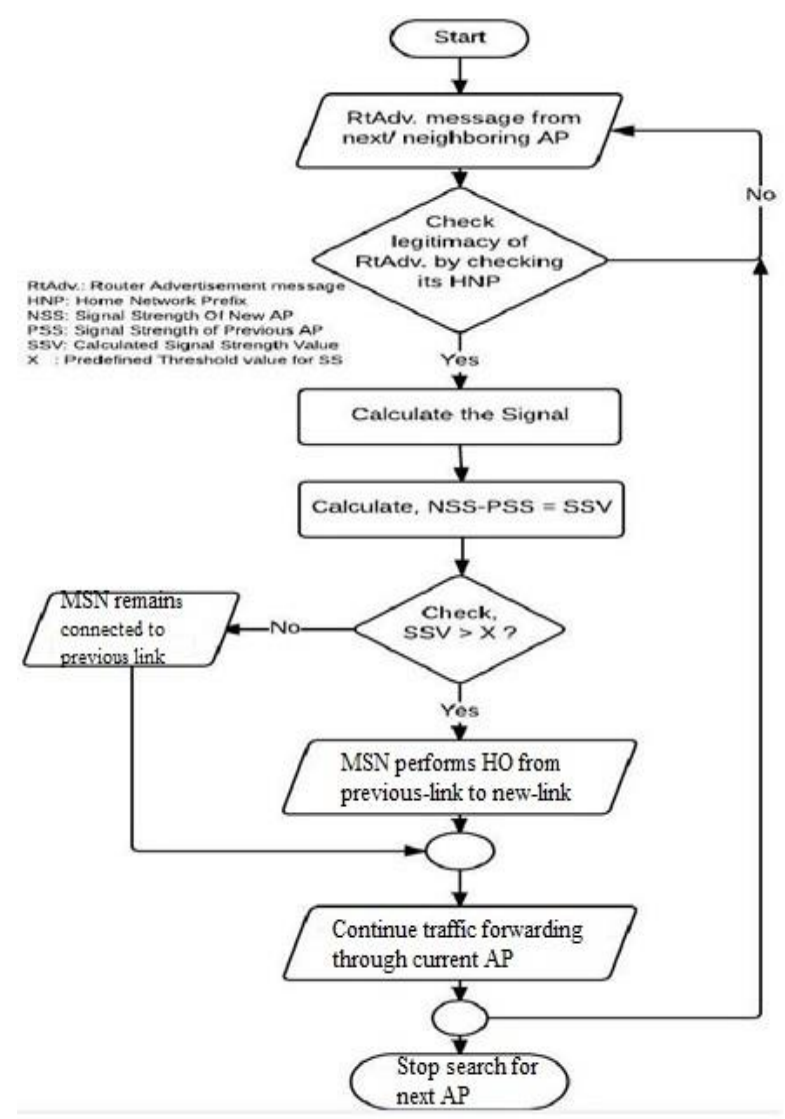

Fig.3. Connection quality check algorithm

\section{A. 6LoWPAN Mobility HO:}

Network-based schemes are actively used in controlled and lossy 6LoWPAN WSN networks [32]. The reason of their extensive usage over host-based schemes is due to introduction of new network entities. These network entities are Local mobility anchor (LMA) and MAG. LMA is responsible for reachability state of MSN whereas MAG performs signaling on behalf of MSN and relieves it from resource consumption. The basic network architecture for network-based PMIPv6 and its signaling flow diagram is shown in fig. 4(a) and fig. (b).

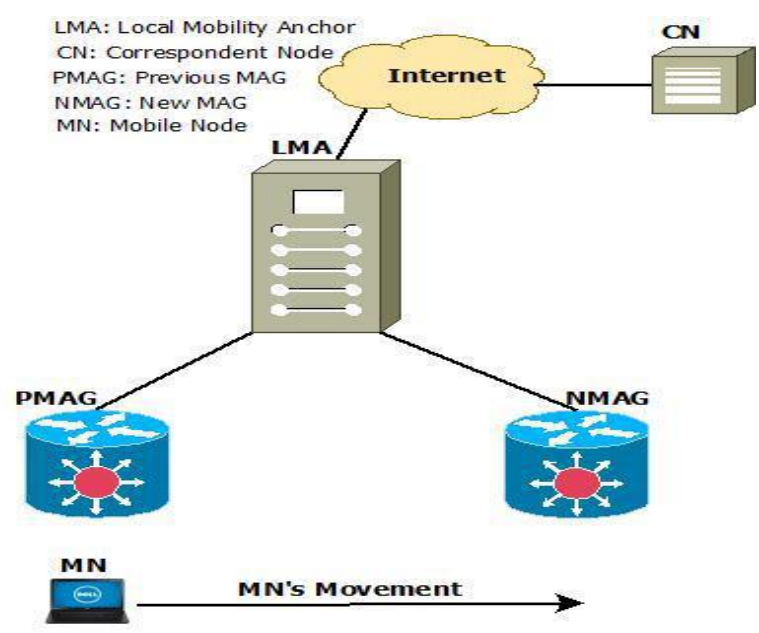

Fig.4.(a) PMIPv6 basic architecture 


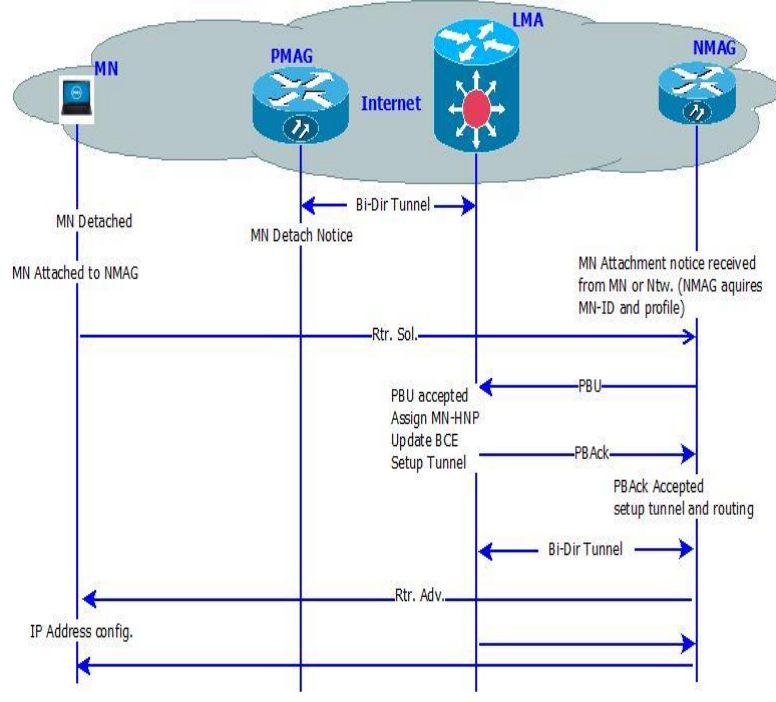

Fig.4.(b) PMIPv6 signaling flow

The HO procedure for PMIPv6 is carried out in the following steps (see fig. 4(b)):

1) The MN disconnects from the PMAG and attaches to NMAG.

2) The MN sends a router solicitation (Rtr Sol) message to NMAG.

3) The NMAG sends the proxy binding update (PBU) message to the MN's LMA.

4) Upon accepting the PBU message, the LMA allocates a mobile node's home network prefix (MNHNP), set up a binding cache entry (BCE) and set up a tunnel between itself and the NMAG.

5) The LMA sends the proxy binding acknowledgement (PBAck) message including the MNHNP to the NMAG.

6) Upon receiving the PBAck message, the NMAG sets up a tunnel to the LMA and the routing for MN's traffic.

7) A bi-directional tunnel between the LMA and NMAG is established.

8) The NMAG sends the router advertisement (RA) message to the $\mathrm{MN}$ on the access link to advertise the MN's HNP.

9) Upon receiving the RA message, the $\mathrm{MN}$ configures its IP address.

10) Correspondent node $(\mathrm{CN})$ communicates with the MN via LMA and NMAG.

\section{B. PMIPv6 HO Analysis:}

HO in PMIPv6 is performed through network entities LMA and MAG. There are many factors which cause delay and are responsible for excessive signaling cost and packet loss. Packet loss in PMIPv6 operations occur due to the connection termination in PMIPv6 protocol during $\mathrm{HO}$ and excessive signaling cost is due to the longer registration process. Many delay factors are there in registration process which constitutes $\mathrm{HO}$ latency. We have analyzed HO latency and packet loss occurring due to extra signaling cost in equations (1) and (2) respectively. For the notations used in these equations, refer table 2 .

Table 2. Notations used for theoretical analysis

\begin{tabular}{|l|l|}
\hline Parameter & Description \\
\hline$D_{\text {L2 }}$ & Layer 2 delay \\
\hline$D_{\text {RS }}$ & Delay occurred for sending Router Solicitation message \\
\hline$D_{\text {MN-Profile }}$ & Delay occurred for obtaining the MN's profile \\
\hline$D_{\text {PBU }}$ & Delay occurred for sending PBU \\
\hline$D_{\text {HNP,BCE,LMA=MAG }}$ & Delay occurred for allocating HNP, creating BCE and establishing tunnel between LMA and MAG \\
\hline$D_{\text {PBA }}$ & Delay occurred for sending back the acknowledgement of PBU \\
\hline$D_{\text {RA }}$ & Delay occurred for advertising RA message on access link \\
\hline$D_{\text {NMAG=MN }}$ & Delay for which the packet remains inside the tunnel between NMAG and MN \\
\hline$\lambda$ & Data traffic rate in packets per unit time \\
\hline$P_{\text {L }}$ & Packet length in octets or byte \\
\hline$n$ & Number of Sensors if group mobility is considered \\
\hline PL_Proposed & Packet loss during handover due to untimely HO prediction in proposed scheme \\
\hline \multicolumn{1}{|c|}{ HO-latency_Proposed } & Handoff latency caused by signaling messages for proposed scheme \\
\hline$D_{\text {PAR-AP }}$ & Delay between previous access router and its AP \\
\hline$D_{\text {PAR_NAR }}$ & Delay that MSN experiences while moving from PAR to NAR in proposed scheme \\
\hline$D_{\text {U_Pred }}$ & Delay for untimely HO prediction in proposed scheme \\
\hline
\end{tabular}

HO-Latency: Let PMIPv6 $6_{\text {HO-Latency }}$ denotes the HO latency in PMIPv6 protocol. This HO latency is caused by various delay factors and can be expressed as:

$$
\begin{gathered}
\text { PMIPv6 } 6_{\text {HO-Latency }}=D_{\mathrm{L} 2}+D_{\mathrm{RS}}+\mathrm{D}_{\mathrm{MN}-\text { Profile }}+\mathrm{D}_{\mathrm{PBU}}+ \\
\mathrm{D}_{\mathrm{HNP}, \mathrm{BCE}, \mathrm{LMA}=\mathrm{MAG}}+\mathrm{D}_{\mathrm{PBA}}+\mathrm{D}_{\mathrm{RA}}+\mathrm{D}_{\mathrm{NMAG}=\mathrm{MN}}
\end{gathered}
$$

Packet Loss: During PMIPv6 HO packets are dropped from PMAG as connection terminates due to movement to new domain/AP. This packet loss can be calculated as product of data traffic rate $(\lambda)$, packet length $\left(\mathrm{P}_{\mathrm{L}}\right)$ and probability of packet loss. The maximum probability of packet loss is determined during the time when $\mathrm{HO}$ is taking place (i.e. disconnection from previous link and connection to new link). Let PMIPv6 $6_{\mathrm{PL}}$ denotes the packet 
loss in PMIPv6 protocol, it can be expressed as:

$$
\begin{gathered}
\text { PMIPv6 }_{\mathrm{PL}}=\lambda \cdot \mathrm{P}_{\mathrm{L}} \cdot\left(\mathrm{D}_{\mathrm{L} 2}+\mathrm{D}_{\mathrm{RS}}+\mathrm{D}_{\mathrm{MN}-\mathrm{Profile}}+\mathrm{D}_{\mathrm{PBU}}+\right. \\
\left.\mathrm{D}_{\mathrm{HNP}, \mathrm{BCE}, \mathrm{LMA}=\mathrm{MAG}}+\mathrm{D}_{\mathrm{PBA}}+\mathrm{D}_{\mathrm{RA}}\right)
\end{gathered}
$$

In the equation (2), $\mathrm{D}_{\mathrm{NMAG}=\mathrm{MN}}$ is not included in packet loss probability part as it is the time when tunnel is established between NMAG and MN. After tunnel establishment packets are delivered to $\mathrm{MN}$.

\section{Proposed Mobility Algorithm:}

Our proposed mobility algorithm is based on advanced $\mathrm{HO}$ prediction, combining L2, L3 HO with prior address configuration. The algorithm is aimed at reducing the signaling cost, HO delay and packet loss. The idea behind our solution is based on:

1) A HO can be predicted and new access router (NAR) is triggered to send HO-request to Domain Gateway Router (DGR) so that downlink traffic from DGR to MSN could be switched to the NAR before HO initiation. The NAR will buffer the traffic until MSN is connected after HO.

2) Downlink traffic arriving at previous access router (PAR) will be buffered if the MSN has already disconnected and this traffic is redirected to NAR after HO completion through bidirectional tunnel. The NAR will inform PAR about $\mathrm{HO}$ completion and will instruct PAR to redirect buffered traffic. The induced buffering mechanism reduces packet loss up to some extent.

3) $\mathrm{L} 3 \mathrm{HO}$ is performed by using $\mathrm{L} 2 \mathrm{HO}$-info message simultaneously for prior address configuration. This address configuration is done before actual movement thus saves registration time and improves HO latency.

Fig. 5 presents the network model for proposed scheme. Two 6LoWPAN domains are considered where domain 1 contains one PAN and domain 2 contains two PANs. One coordinating MSN with sensing devices is considered. The coordinating MSN makes inter-domain as well as intra-domain movements. During inter-domain movement coordinating MSN changes its HNP through associate node and achieves GDR2's HNP whereas it does not require any change in its HNP when intra-domain movement is made. Intra-domain movement between AR2 and AR3 under same GDR2's HNP can be supported only by changing MSN's interface ID. For inter-domain movement, AR1 acts as PAR and AR2 as NAR. For intra-domain movement, AR2 acts PAR and AR3 as NAR.

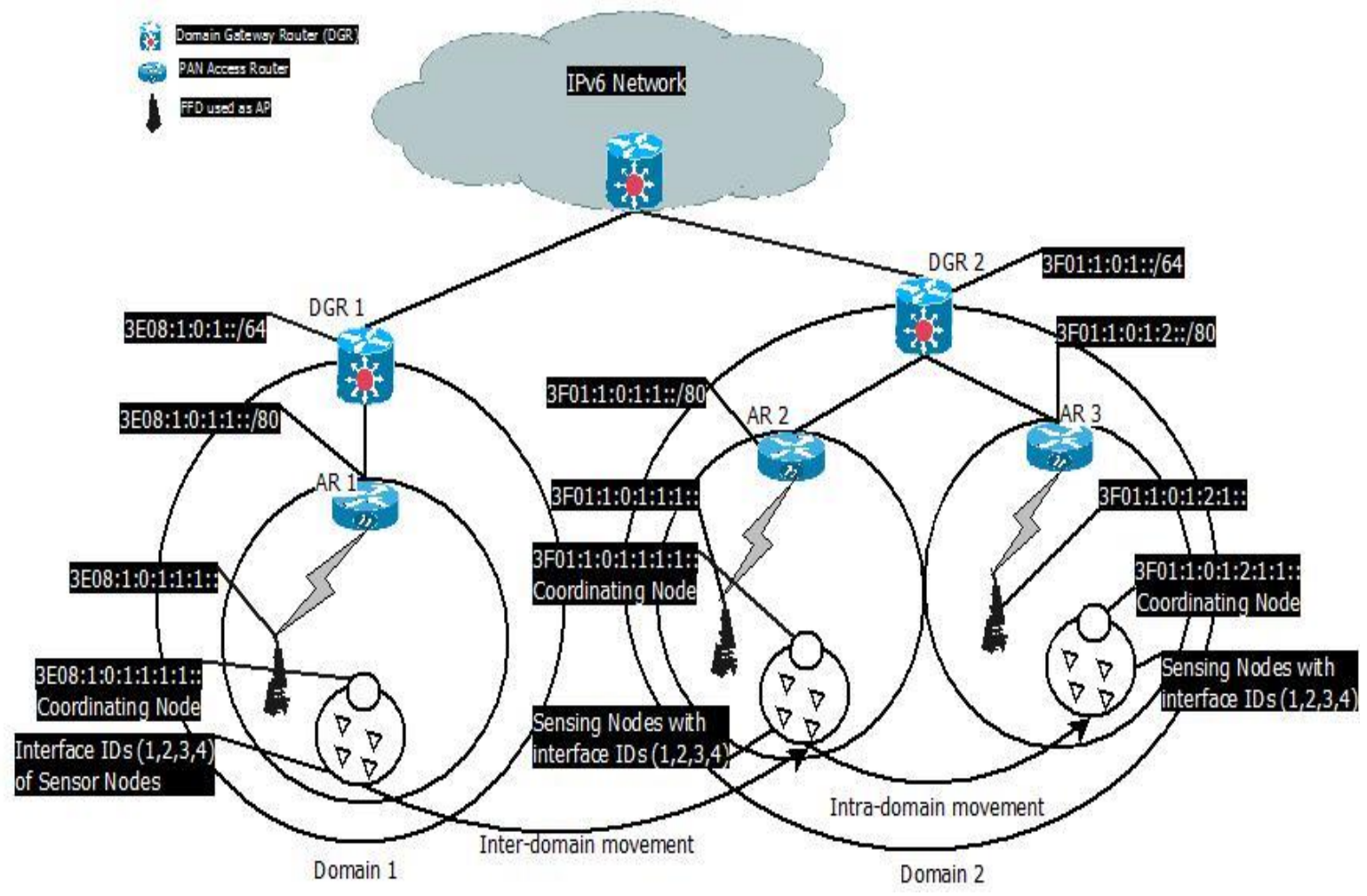

Fig.5. Network model for proposed scheme

\section{HO procedure for proposed scheme:}

Group mobility of sensors supported by a coordinating MSN is shown in fig. 5. The coordinating MSN makes movement based on advanced $\mathrm{HO}$ prediction by using connection quality check algorithm. The HO procedure with signaling flow diagram is shown in fig. 6 .

The HO procedure is initiated prior to the actual disconnection from PAR's link. The HO-registration process is completed first and then MSN disconnects from previous link, performing simultaneous L2, L3 HO. 


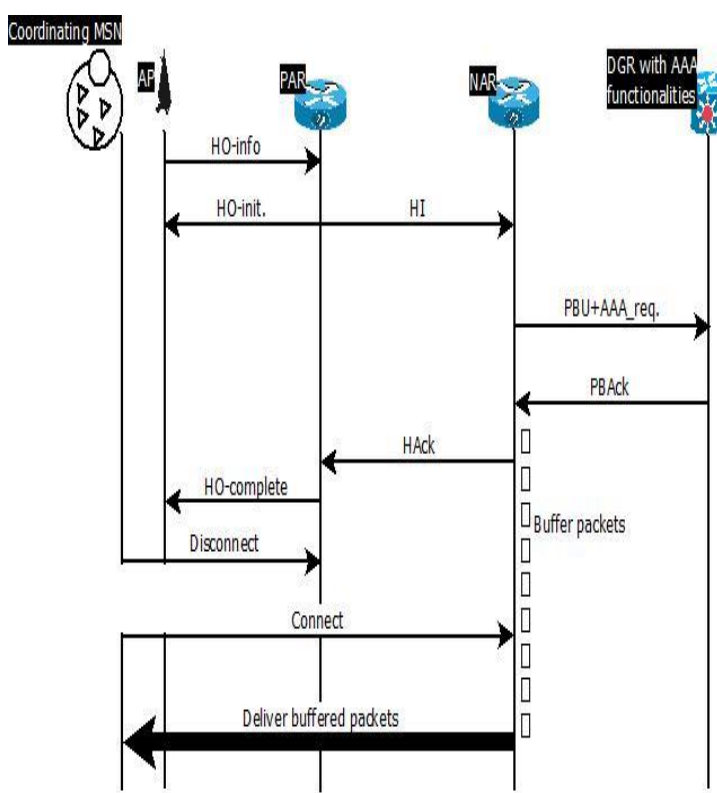

Fig.6. Signaling flow diagram of proposed scheme

For intra-PAN HO, MSN only changes interface ID and HNP remains same. Routing of packets is achieved through globally identified HNP. The HO-procedure for the proposed scheme is presented by the following steps:

1) The PAR receives a periodic notification from $L 2$ that a HO is imminent by checking the RSS value. This L2 notification (HO-info) contains HNP and profile identifier (new access network ID) of predicted NAR to which MSN wants to connect. L2 notification also contains MSN's current ID and profile identifier. The profile identifiers of NAR are broadcasted by PAR's AP on pilot channels as each $\mathrm{AR}$ is equipped with neighbor look up table.

2) PAR derives the address of NAR by using HO-info message.

3) PAR decides to initiate $\mathrm{HO}$ and informs its AP by sending back HO-init message. At the same time PAR also sends handoff initiate (HI) request containing MSN's identifiers along with time stamp to NAR.

4) Upon receiving $\mathrm{HI}$ message, NAR sends BU message with time stamp to DGR. DGR checks the legitimacy of MSN identifiers received in PBU by using AAA-server functionalities. A Proxy binding acknowledgement (PBAck) in response to $\mathrm{PBU}$ is sent back to NAR.

5) Upon receiving the PBAck message, NAR sends handoff acknowledgement (HAck) message to PAR and starts buffering the traffic intended for MSN.

6) PAR sends L2 HO-complete message to its AP to inform MSN about HO. MSN disconnects from PAR's AP and connects to NAR's AP, if it has not done already. If MSN has already moved to the NAR's domain then buffering of traffic takes place at PAR and after $\mathrm{HO}$ completion it is redirected to NAR.

7) After joining the NAR's AP, MSN sends neighbor discovery protocol (NDP) request to NAR and starts receiving the buffered traffic.
The above procedure works well for intra as well as inter domain HO. During intra-domain HO, HNP remains same whereas it changes for inter-domain HO.

\section{E. Performance Analysis of proposed scheme:}

In general, ideal situation is analyzed for performance of proposed scheme while as simulation situation can be little complex. Our solution is aimed at reducing signaling cost, packet loss and HO latency. Extra signaling cost and longer registration delay are responsible for packet loss and HO latency. Therefore we analyze HO latency and packet loss in terms of signaling cost.

HO-Latency: HO-latency is caused by longer registration delay. This registration delay is caused by signaling messages required for registration process. Let HO-Latency_Proposed denotes the HO-latency in proposed scheme and it can be expressed as (for notations used, refer table 2):

$$
\text { HO-latency_Proposed }=\mathrm{D}_{\mathrm{L} 2}+\sum_{\mathrm{k}=1}^{\mathrm{n}} \mathrm{k}\left(\mathrm{D}_{\mathrm{PBU}}+\mathrm{D}_{\mathrm{PBAck}}\right)
$$

For " $n$ " number of mobile sensors, we can rewrite equation (3) as:

$$
\text { HO-latency_proposed }=\mathrm{D}_{\mathrm{L} 2}+\mathrm{n}\left(\mathrm{D}_{\mathrm{PBU}}+\mathrm{D}_{\mathrm{PBAck}}\right)
$$

Average HO-latency is denoted as:

$$
\begin{gathered}
\text { Avg.-HO-latency_Proposed }=1 / \mathrm{n}(\text { HO-latency_Proposed } \\
=\mathrm{D}_{\mathrm{L} 2} / \mathrm{n}+\mathrm{D}_{\mathrm{PBU}}+\mathrm{D}_{\mathrm{PBAck}}
\end{gathered}
$$

In the above equations (3) and (4), only three delay factors are included. $\mathrm{D}_{\mathrm{L} 2}$ is the layer $2 \mathrm{HO}$ comprised of $\mathrm{HO}$ - info, $\mathrm{HI}$, HAck and HO-complete messages. $\mathrm{D}_{\mathrm{PBU}}$ and $\mathrm{D}_{\mathrm{PBAck}}$ include legitimacy check and HNP allocation in new domain. After $P_{B A c k}$ message packets start buffering at NAR, delivered after $\mathrm{HO}$ completion, so rest delay factors cannot be included in HO latency.

Packet Loss: Our proposed scheme is based on advanced HO prediction. There will be no packet loss if HO prediction happens timely. The packets arriving at PAR will get delivered to MSN before its disconnection. NAR will also buffer packets until MSN connects to its access link, therefore prevents packet loss. In case of untimely HO prediction, packets sent from PAR to its AP before PAR gets notified about MSN's disconnection are lost. This packet loss is mainly due to the delay between PAR and its AP. Let PL_proposed denotes packet loss in the proposed scheme. This packet loss can be calculated and expressed as:

$$
\mathrm{PL}_{- \text {proposed }}=\lambda \cdot \mathrm{P}_{\mathrm{L}} \cdot\left[\sum_{\mathrm{k}=1}^{\mathrm{n}} \mathrm{K}\left(\mathrm{D}_{\text {PAR-AP }}\right)+\mathrm{D}_{\mathrm{U}_{-} \text {Pred }}+\right.
$$

For " $n$ " number of sensors, the above equation can be rewritten as:

$$
\left.\mathrm{PL}_{- \text {proposed }}=\lambda \cdot \mathrm{P}_{\mathrm{L}} \cdot\left[\mathrm{n}\left(\mathrm{D}_{\mathrm{PAR}-\mathrm{AP}}\right)+\mathrm{D}_{\mathrm{U}_{-} \text {Pred }}+\mathrm{D}_{\text {PAR-NAR }}\right)\right]
$$


From the theoretical analysis shown above, we find the proposed scheme has less signaling cost when compared to traditional PMIPv6. Less signaling cost leads to shorter registration delays which ultimately reduces $\mathrm{HO}$ latency. Also when we compare our analysis with that of "Ref [33]", a noticeable reduction in signaling cost can be seen. We compare our analysis with existing scheme in [33] due to the following reasons:

1) "Ref [33]" uses network based PMIPv6 protocol instead MIPv6 to achieve mobility support in 6LoWPAN WSN.

2) Group mobility of sensors was observed which can be observed as individual sensor mobility with coordinating MSN as well.

\section{Simulation}

NS-2 simulator on LINUX platform is used to validate our analysis. We studied the simulation scenario presented in fig. 5 for intra as well as inter domain mobility in 6LoWPAN. The various parameters used are shown in table 3.

The simulation process was carried out by building network model and implementing computer program for the proposed scheme. Performance metrics were observed depending upon behavior of proposed scheme.

Table 3. Parameters used in simulation

\begin{tabular}{|l|l|}
\hline Parameter & Parameter value \\
\hline Simulation model & Random waypoint \\
\hline Simulation area & $500 \times 500 \mathrm{~m}^{2}$ \\
\hline PAR-NAR distance (intra-domain) & $100 \mathrm{~m}$ \\
\hline AP coverage range & $50 \mathrm{~m}$ radius \\
\hline FFD used & 9 \\
\hline RFD used & 4 \\
\hline MAC protocol & IEEE 802.15 .4 \\
\hline Packet size & $1000 \mathrm{bytes}$ \\
\hline Data rate & $250 \mathrm{kbps}$ \\
\hline Wireless link delay & $10 \mathrm{~ms}-100 \mathrm{~ms}$ \\
\hline Speed & $1 \mathrm{~m} / \mathrm{s}-10 \mathrm{~m} / \mathrm{s}$ \\
\hline Simulation time & $500 \mathrm{~s}$ \\
\hline
\end{tabular}

\section{A. Results and Discussion:}

Results for different performance metrics such as signaling cost, HO latency and packet loss were taken. In the first round these metrics were observed by varying the speed of MSN from $1 \mathrm{~m} / \mathrm{s}$ (walking speed) to $10 \mathrm{~m} / \mathrm{s}$. In the second round, wireless link delay (WLD) is varied from $10 \mathrm{~ms}$ to $100 \mathrm{~ms}$ to observe the same parameters. Initially the results for intra-domain mobility $\mathrm{HO}$ were taken followed by the inter-domain mobility HO.

Intra-domain mobility: intra-domain mobility takes place between AR2 and AR3. MSN doesn't need to change its HNP and only interface ID of MSN changes. Communication in intra-domain $\mathrm{HO}$ is achieved through routing backbone. The HO delay, signaling cost and packet loss occurring due to intra-domain mobility $\mathrm{HO}$ based on speed and WLD are shown in figs. 7-9.

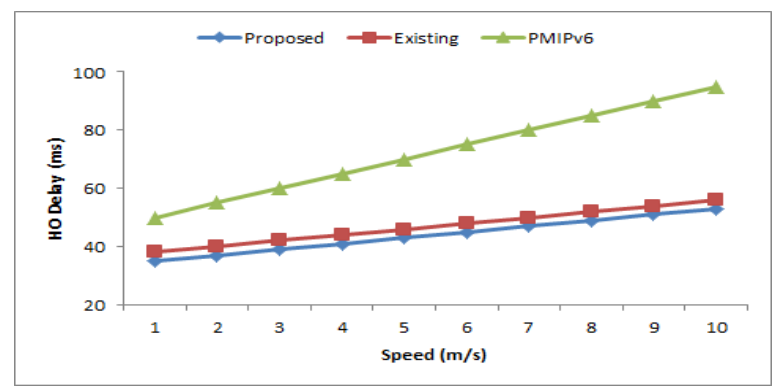

(a) HO delay Vs MSN speed

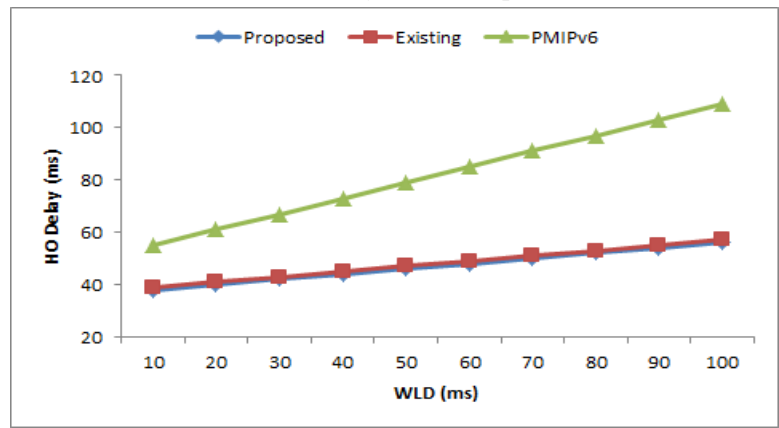

(b) HO delay Vs WLD

Fig.7. HO delay based on (a) MSN speed and (b) WLD (intra-domain)

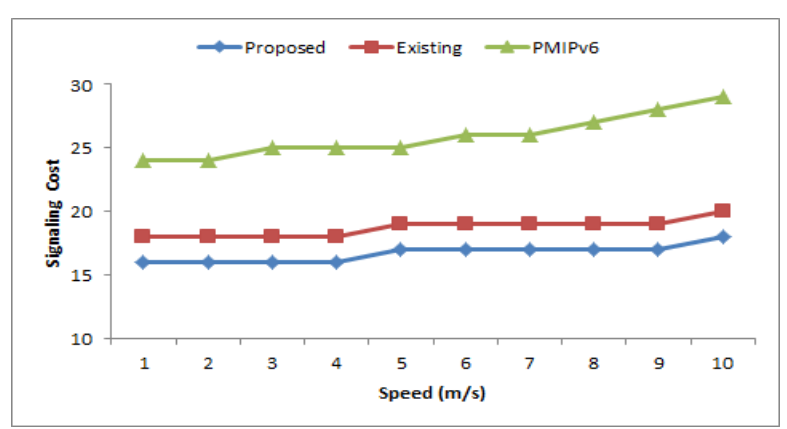

(a) Signaling cost Vs MSN speed

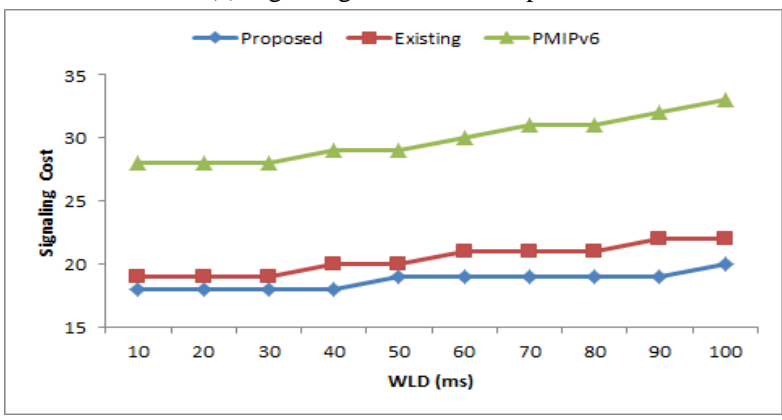

(b) Signaling cost Vs WLD

Fig.8. Signaling cost based on (a) MSN speed and (b) WLD(intradomain) 


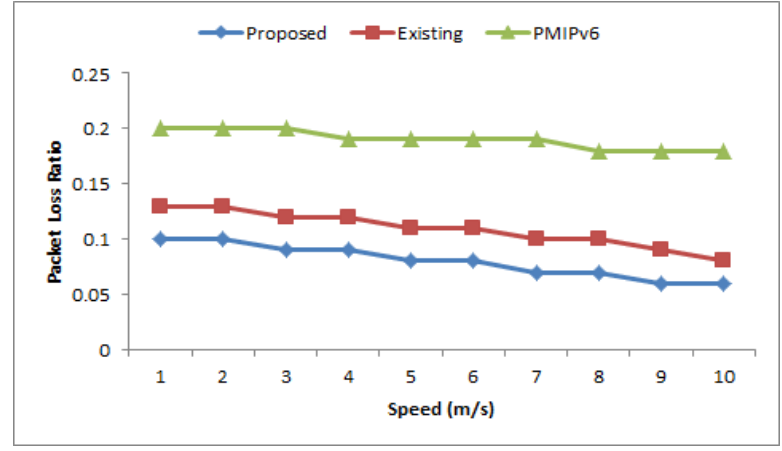

(a) Packet loss ratio Vs MSN speed

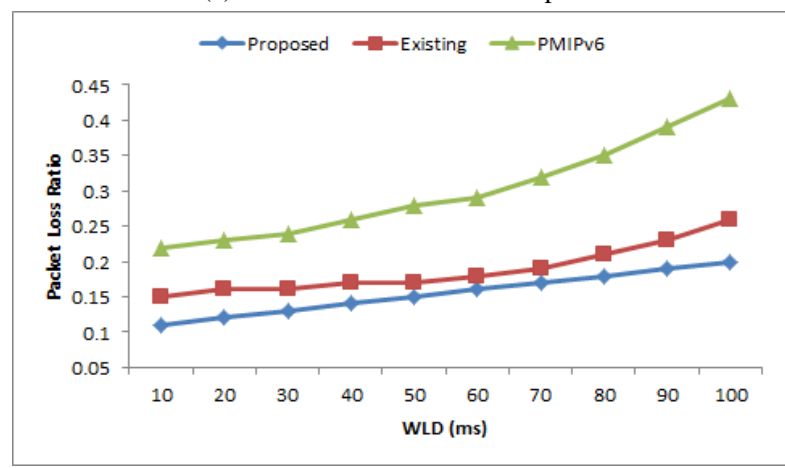

(b) Packet loss ratio Vs WLD

Fig.9. Packet loss ratio based on (a) MSN speed and (b) WLD(intradomain)

With the increase in MSN speed, there is slight increase in $\mathrm{HO}$ delay of all the three schemes. This increase is due to the link instability and increased probability of frequent HOs. With the frequent HOs, MSN registers again and again with new APs and thus increases signaling cost. This signaling cost adds up additional HO delay. Increased WLD increases HO delay because higher link delay suffers high $\mathrm{HO}$ and transmission latencies. The increased WLD is considered as one parameter because of other factors such as signal fading, attenuation, network congestion etc. Signaling cost in the intra-domain mobility does not vary much due to exchange of limited number of message for registration purpose in the same domain. Also the increased WLD does not affect signaling cost much. Packet loss ratio based on MSN speed shows a slight decrease because in intra-domain mobility, initially there would be some loss of packets due to untimely $\mathrm{HO}$ prediction. Once $\mathrm{HO}$ prediction is made, buffering mechanism gets active and reduces packet loss. With increased WLD, packet loss increases slightly due to high transmission latencies.

Inter-domain mobility: Inter domain mobility takes place between AR1 and AR2/AR3. During inter domain $\mathrm{HO}$, both HNP and interface ID change. Routing of packets achieved through newly attained HNP and then sent to desired interface ID. Results for HO delay, signaling cost and packet loss in inter domain mobility are presented in figs. 10-12.

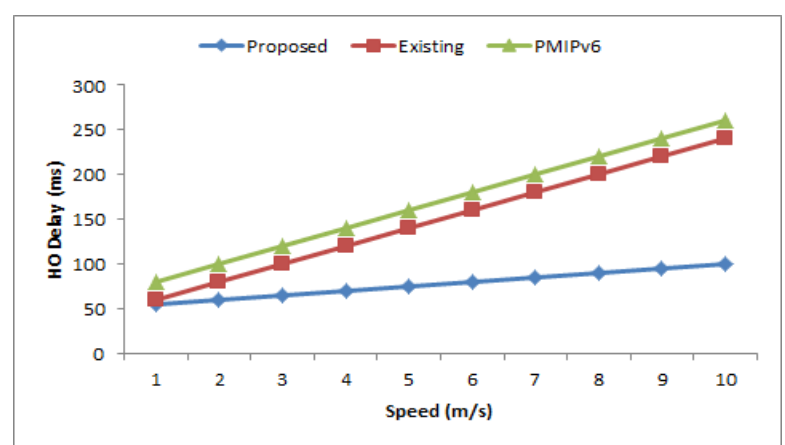

(a) HO delay Vs MSN speed

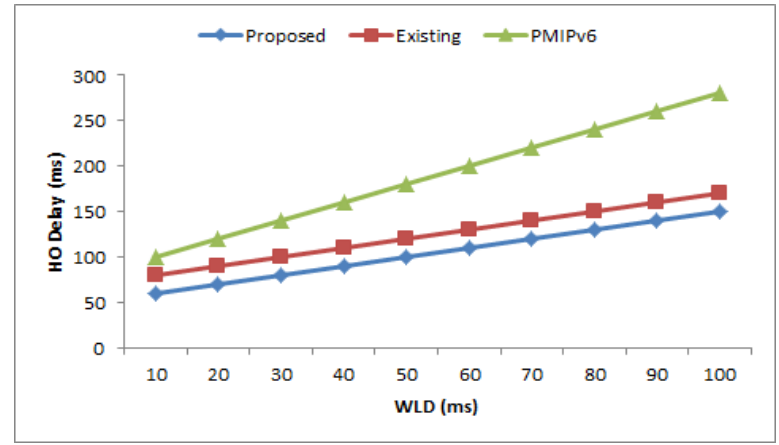

(b) HO delya Vs WLD

Fig.10. HO delay based on (a) MSN speed and (b) WLD (inter-domain)

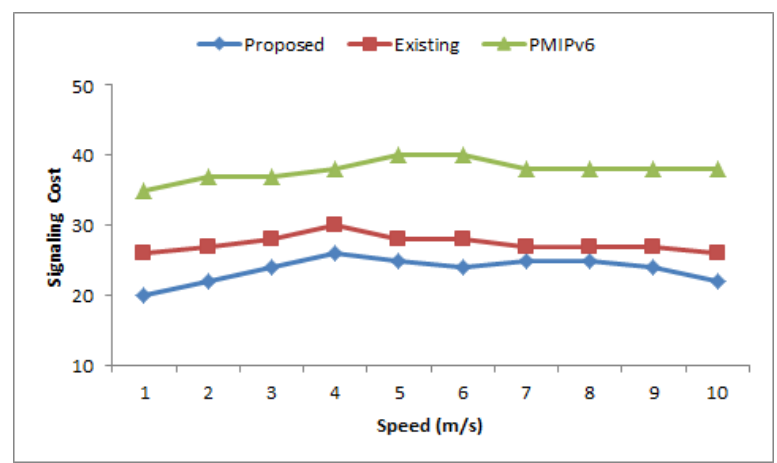

(a) Signaling cost Vs MSN speed

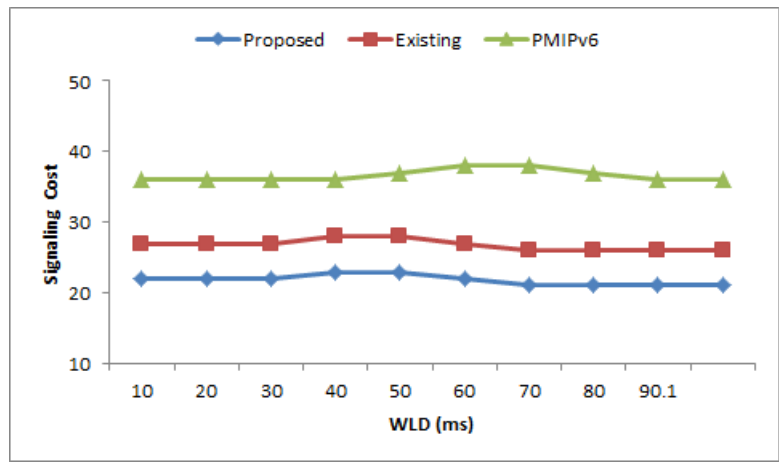

(b) Signaling cost Vs WLD

Fig.11. Signaling cost based on (a) MSN speed and (b) WLD (interdomain) 


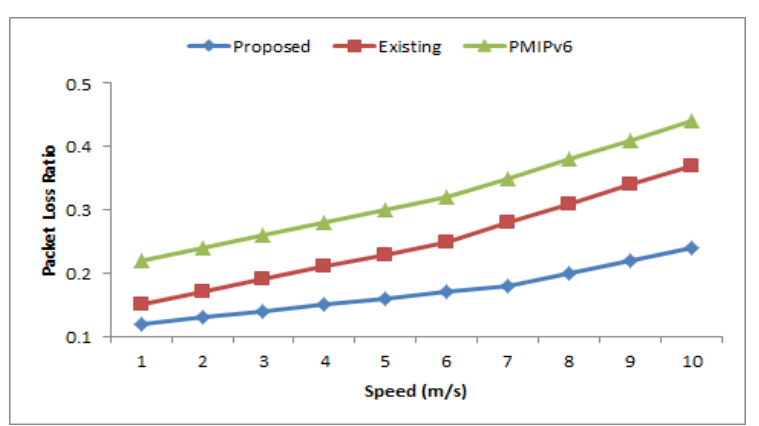

(a) Packet loss ratio Vs MSN speed

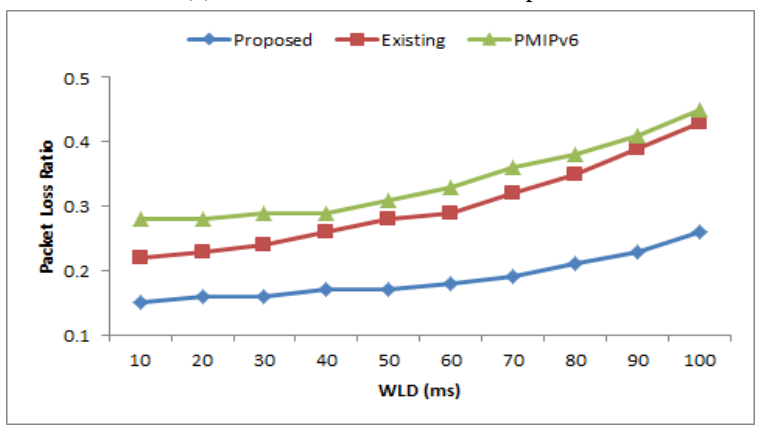

(b) Packet loss ratio Vs WLD

Fig.12. Packet loss ratio based on (a) MSN speed and (b) WLD (interdomain)

With the increase in MSN speed, inter-domain $\mathrm{HO}$ delay is less for proposed scheme when compared to existing and PMIPv6 schemes. The increased HO delay for existing and traditional PMIPv6 scheme is due to their longer registration process. With increase in WLD, existing scheme does not vary much with respect to proposed but the PMIPv6 shows increase in HO delay due to high transmission latency. Signaling cost for all the three schemes shows similar results based on MSN speed and WLD. It is more for PMIPv6 and existing scheme due to exchange of extra signaling messages such RS and RA. Packet loss in proposed scheme occurs due to untimely $\mathrm{HO}$ prediction or failed $\mathrm{HO}$ prediction only and it is quite less when compared to existing and PMIPv6 schemes. Other two schemes suffer packet loss due to the connection termination during HO.

In general, when we compare intra-domain and interdomain $\mathrm{HO}$ performance, we find more improvements in intra-domain HO than inter-domain. Proposed scheme performs better in both types of HOs. The main reasons are analyzed as follows:

1) Advanced $\mathrm{HO}$ prediction by using connection quality check algorithm.

2) Buffering mechanism to prevent packet loss.

3) Simultaneous L2 and L3 HO occurrence without channel scanning and prior auto address configuration before actual movement.

\section{CONCLUSION}

In this paper we have observed the current solutions for 6LoWPAN WSN mobility. Based on our observations, HO delay is mainly caused by extra signaling cost and longer registration delay. Channel scanning in L2 $\mathrm{HO}$ is most time consuming and L3 HO poses longer address registration delay. Packet loss incurs due to connection termination during $\mathrm{HO}$ and longer $\mathrm{HO}$ latencies. Therefore, we are motivated to propose a mobility management scheme based on advanced HO prediction with prior address registration. Also the buffering mechanism reduces the packet loss incurs during HO. L2 and L3 HO is performed simultaneously without channel scanning. The proposed scheme is analyzed and validated through simulation using ns- 2 tool. From the results it is observed that the proposed scheme improves $\mathrm{HO}$ performance in intra-domain and inter-domain 6LoWPAN mobility.

\section{ACKNOWLEDGMENT}

This work was supported by National Institute of Technology (NIT) Srinagar, India.

\section{REFERENCES}

[1] Julien Montavont, Damien Roth, Thomas Noël. Mobile IPv6 in internet of things: analysis, experimentations and optimizations. Jr. of Ad Hoc Networks, March 2014, Volume 14, pp. 15-25.

[2] M. Singh, S. Singh, P. Pancholi, N. Saxena, R. K. Mehrotra. Modeling of machine to machine communication networks. In Proc. ICT, 2013, pp. 258162.

[3] D. Miorandi, S. Sicari, F. De Pellegrini, I. Chlamtac. Internet of things: Vision, applications and research challenges. Journal of Ad Hoc Networks, 2012, pp. 14971516.

[4] D. Johnson, C. Perkins, J. Arkko. Mobility support in IPv6. IETF RFC 3775, Internet engineering task force, June 2004.

[5] Gundavelli, S.; Leung, K.; Devarapalli, V.; Chowdhury, K.; Patil, B. Proxy mobile IPv6. August 2008, RFC 5213.

[6] Vasaka Visoottiviseth, Pairat Ngamtura. On the Performance of MIPv6 and FMIPv6 based on real IPv6 applications over IEEE $802.11 \mathrm{~g}$ Testbeds. Int. Symposium on Commun. and Inf. Technol. (ISCIT), Oct. 2010, pp. 1217-1222.

[7] Riaz A Khan, A H Mir. A Simulation Based Study of IP Mobility over IPv6 Networks. In Proc. Int. conf. Innovative App. of Comput. Intelligence on Power, Energy and Controls with their Impact on Humanity (CIPECH14), Nov. 2014, pp. 196-201.

[8] Myung-Kyu Yi, Jin-Woo Choi, Young-Kyu Yang. A comparative analysis on the signaling load of proxy mobile IPv6 and hierarchical mobile IPv6. In Proc. 4th IEEE Int. Symposium on Wireless Pervasive Computing (ISWPC), Feb. 2009, pp. 1-5.

[9] Jinho Kim, R. Haw, Eung Jun Cho, Choong Seon Hong, Sungwon Lee. A 6LoWPAN sensor node mobility scheme based on proxy mobile IPv6. IEEE Transactions on Mobile Computing, Dec. 2012, vol.11, no.12, pp. 20602072.

[10] R. Silva, J. S. Silva, F. Boavida. A proposal for proxybased mobility in WSNs. Journal of Comp. Commun. 2012, Vol. 35, no. 10, pp. 1200-1216.

[11] Y. Li, Y. Jiang, H. Su, D. Jin, L. Su, L. ZengA. A group based handoff scheme for correlated mobile nodes in proxy mobile IPv6. In Proc. Global Telecommunication Conf., Dec. 2009, pp. 1-6. 
[12] Islam MM, Huh EN. Sensor proxy mobile IPv6 (SPMIPv6) - a novel scheme for mobility supported IPWSNs. Sensors 2011, 11(2), pp. 1865-1887.

[13] Xiaonan Wang, Hongbin Cheng. Research on seamless mobility handover for 6LoWPAN wireless sensor networks. Telecommun Syst 2015, pp. 1-17.

[14] Oliveira LM, De Sousa AF, Rodrigues JJ. Routing and mobility approaches in IPv6 over LOWPAN mesh networks. Int. J. Commun. Syst. 2011, Vol. 24, no. 11, pp. 1446-1466.

[15] Soliman H, Castellucia C, El Malki K, Bellier L. Hierarchical mobile IPv6 mobility management (HMIPv6). Aug. 2005, RFC 4140.

[16] Jabir AJ, Subramaniam SK, Ahmed ZZ, et al. A cluster based proxy mobile IPv6 for IP-WSNs. EURASIP J. Wireless Commun. Netw. 2012, vol. 1, pp. 1-17.

[17] Akyildiz IF, Xie J., Mohanty S. A survey of mobility management in next-generation all-IP-based wireless systems. IEEE trans. Wireless Communications, Aug. 2004, Vol. 11, no. 4, pp. 16-28.

[18] Kempf J. Problem statement for network-based localized mobility management. April 2007, RFC 4830.

[19] Ping Dong, Hongke Zhang, Hongbin Luo, Ting-Yun Chi, Sy-Yen Kuo. A network-based mobility management scheme for future Internet. Computers \& Electrical Engineering, March 2010, Volume 36, Issue 2, pp. 291 302.

[20] Bag G, Raza M T, Kim K H, Yoo S W. LowMob: Intra PAN mobility support schemes for 6LoWPAN. Sensors, July 2009, Vol. 9, no.7, pp. 5844-5877.

[21] Minkeun Ha, Daeyoung Kim, Seong Hoon Kim, Sungmin Hong. Inter-MARIO: A fast and seamless mobility protocol to support Inter_PAN handover in 6LoWPAN. In Proc. Global Telecommunications Conference (GLOBECOM), Dec. 2010, pp. 1-6.

[22] Juha Petajajarvi, Heikki Karvonen. Soft handover for mobile wireless sensor networks based on 6LoWPAN. In Proc. Int. Conf. Distributed Computing in Sensor Systems and Workshops (DCOSS), June 2011, pp. 1-6.

[23] Sergio Gonzalez, Min Chen, Leung V.C.M. Mobility support for health monitoring at home using wearable sensors. IEEE trans. Information technology in Biomedicine, July 2011, Vol. 15, No. 4, pp. 539-549.

[24] Md. Motaharul Islam, Mohammad Mehedi Hassan, EuiNam Huh. Sensor proxy mobile IPv6 (SPMIPv6) - A framework of mobility supported IP-WSN. In Proc. 13th Int. Conf. Computer and Information Technology (ICCIT), Dec. 2010, pp. 295-299.

[25] Jinho Kim, Haw R., Eung Jun Cho, Choong Seon Hong, Sungwon Lee. A 6LoWPAN sensor node mobility scheme based on proxy mobile IPv6. IEEE Transactions on Mobile Computing, Dec. 2012, vol.11, no.12, pp. 20602072.

[26] Sofiane Imadali, Karanasiou A., Petrescu A., Sifniadis I., Veque V., Angelidis P. EHealth service support in IPv6 vehicular networks. In Proc. 8th Int. Conf. wireless and mob. comput. networking and communications (WiMob), Oct. 2012, pp. 579-585.

[27] Shahamabadi M.S., Bin Mohd Ali B., Varahram P., Jara A. J. A network mobility solution based on 6LoWPAN hospital wireless sensor network (NEMO-HWSN). In Proc. 7th Int. Conf. Innovative Mobile and Internet Services in Ubiquitous Computing, July 2013, pp. 433438.

[28] Cheng-Wei Lee, Meng Chang Chen, Yeali S. Sun. A novel network mobility management scheme supporting seamless handover for high-speed trains. Computer
Communications, Jan. 2014, Vol. 37, pp. 53-63.

[29] Ricardo Silva, Jorge Sa Silva, Fernando Boavida. Mobility in wireless sensor networks - Survey and proposal, Computer Communications, Volume 52, 1 October 2014, pp. 1-20.

[30] Xiaonan Wang, Qi Sun, Yuan Yang. A cross-layer mobility support protocol for wireless sensor networks, Computers \& Electrical Engineering, Volume 48, November 2015, pp. 330-342.

[31] Wireless Medium Access Control and Physical Layer Specifications for Low-rate Wireless personal area Networks. (Sept. 2006). IEEE Standard 802.15.4, 2003

[32] Khan R.A., Mir A.H. EMS: Enhanced mobility scheme for controlled and lossy networks. In International Conference on Green Computing and Internet of Things (ICGCIoT), 8-10 Oct. 2015, pp. 655-660.

[33] Yuh-Shyan Chen, Chih-Shun Hsu, Hau-Kai Lee. An enhanced group mobility protocol for 6LoWPAN-based wireless body area networks. IEEE trans. Sensors Journal, March 2014, Vol. 14, no. 3, pp. 797-807.

[34] Kim H.S., Park S.H., Park C.S., Kim J.W., Ko S.J. Selective channel scanning for fast handoff for wireless LAN using neighbor graph. In Proc. Int. Conf. Pers. Wireless Commun. Sept.2004, pp. 194-203.

\section{Authors' Profiles}

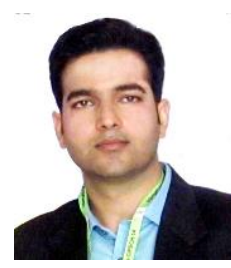

Riaz A. Khan received the B.TECH. degree in computer science engineering from university of Jammu, J\&K, India in 2008 and the M.TECH. degree in Communication and Information Technology from National Institute of Technology (NIT) Srinagar, India in 2012. $\mathrm{He}$ is currently pursuing Ph.D. degree in computer networks at National Institute of Technology, Srinagar, India. His areas of interest are computer networks, wireless sensor networks and network security. He can be contacted at: riazk3@gmail.com.

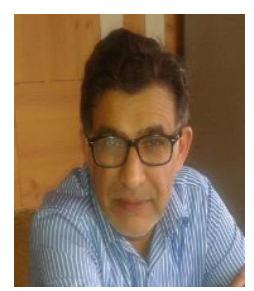

Ajaz Hussain Mir has done his B.E in Electrical Engineering with specialization in Electronics \& Communication Engineering (ECE). He did his M.Tech in Computer Technology and $\mathrm{Ph} . \mathrm{D}$ both from IIT Delhi in the year 1989 and 1996 respectively. He is Chief Investigator of Ministry of Communication and Information Technology, Govt. of India project: Information Security Education and Awareness (ISEA). He has been guiding $\mathrm{Ph} . \mathrm{D}$ and M.Tech thesis in Security and other related areas and has a number of International publications to his credit. Presently he is working as Professor in the Department of Electronics \& Communication Engineering at NIT Srinagar, India. His areas of interest are Biometrics; Image processing, Security, Wireless Communication and Networks.

How to cite this paper: Riaz Ahmed Khan, Ajaz Hussain Mir,"Advanced Prediction Based Mobility Support for 6LoWPAN Wireless Sensor Networks", International Journal of Information Technology and Computer Science(IJITCS), Vol.9, No.2, pp.47-57, 2017. DOI: 10.5815/ijitcs.2017.02.06 